\title{
PRESIÓN DE CAZA EN MAMÍFEROS DE LA RESERVA NACIONAL MATSÉS, AL NORESTE DE LA AMAZONÍA PERUANA
}

Claudio BARDALES-ALVITES ${ }^{1}$, León TORRES-OYARCE ${ }^{1}$, Emérita TIRADO-HERRERA², Miguel ANTÚNEZ-CORREA ${ }^{3}$

1 Facultad de Ciencias Biológicas. Universidad Nacional de la Amazonía Peruana. Av. Elías Aguirre № 1342. Iquitos, Perú. claudiobardales92@gmail.com/leonfernandotorresoyarce@gmail.com

2 Departamento de Ecología y Fauna. Facultad de Ciencias Biológicas. Universidad Nacional de la Amazonía Peruana. Calle Pevas 5 ta cuadra $S / \mathrm{N}$. Iquitos, Loreto-Perú.

3 Instituto del Bien Común. Iquitos, Loreto-Perú. Calle Nauta Nº 334

\section{RESUMEN}

De junio a octubre del 2015, se realizó el estudio en la Reserva Nacional Matsés (RNM), con la finalidad de evaluar la presión de caza de mamíferos. Los datos se obtuvieron de los sectores Alemán y Loboyacu, donde se usaron registros de aprovechamiento de animales de caza, mapas participativos, entrevistas semiestructuradas y diálogos interactivos. El majas Cuniculus paca, fue la especie más cazada en ambos sectores. El área de caza estimada para el sector Alemán fue de $452 \mathrm{~km}^{2}$ y de Loboyacu fue $497 \mathrm{~km}^{2}$. La estrategia de caza más frecuente en ambos sectores fue la búsqueda en canoa y trocha usando escopeta. El conocimiento de la presión de caza ayuda a entender el desarrollo de la cacería, y ayuda a reaccionar anticipadamente a posibles amenazas que podrían estar afectando a las poblaciones de fauna silvestre.

PALABRAS CLAVE: Estrategias de caza, Herramientas de caza, Mamíferos, Presión de caza, Reserva Nacional Matsés.

\section{HUNTING PRESSURE IN MAMMALS OF MATSÉS NATIONAL RESERVE, PERUVIAN NORTHERN AMAZON}

\section{ABSTRACT}

From June to October 2015, the study was carried out in Matses National Reserve (RNM), in order to evaluate the pressure of hunting of mammals. Data were obtained from the Aleman and Loboyacu sectors, where hunting registers, participatory maps, semi-structured interviews and interactive dialogues were used. The paca Cuniculus paca was the most hunted species in both sectors. The estimated hunting area for Aleman sector and Loboyacu sector was $452 \mathrm{~km}^{2}$ and $497 \mathrm{~km}^{2}$ respectively. The most frequent hunting strategies in both sectors were the search in canoe and trail using shotgun. In conclusion, Knowledge of hunting pressure helps to understand the development of the hunt, and helps to react early to potential threats that could be affecting wildlife populations.

KEYWORDS: Hunting pressure, Hunting Strategies, Hunting tools, Mammals, Matses National Reserve. 


\section{INTRODUCCIÓN}

La cacería de animales silvestres es una actividad fundamental para el poblador amazónico, que por décadas ha representado una importante fuente proteica en combinación con el pescado (Ojasti 1993; Bodmer et al., 1999; SERNANP 2014; Aquino \& Calle 2003). Sin embargo, la práctica desmedida de esta actividad trae consigo impactos negativos sobre las poblaciones de animales, provocando disminución en la abundancia y a veces extinciones locales. Por ello es importante conocer aspectos influyentes en la presión de caza, tales como el área donde se realiza la cacería, técnicas y herramientas empleadas y volumen de extracción, que en su conjunto permiten conocer cómo se desarrolla la cacería y detectar cambios que podrían afectar las poblaciones de fauna.

En la Reserva Nacional Matsés se realiza la cacería de subsistencia, al igual que en otra áreas de la Amazonía peruana, como en la Reserva Nacional Pacaya-Samiria (Bodmer et al., 1999; Aquino \& Calle, 2003) en donde los primates son los más cazados, mientras que en el Área de Conservación Regional Comunal Tamshiyacu-Tahuayo (Puertas, 1999; Hurtado-Gonzales \& Bodmer, 2004 ), río Pucacuro (Pérez-Peña et al., 2016), río Algodón (Aquino et al., 2007a), cuenca alta del río Itaya (Aquino et al., 2007b) y la Concesión de Conservación Lago Preto Paredón (Pérez-Peña, 2011)los roedores y ungulados son los más cazados.

La jefatura de la Reserva Nacional Matsés, permite y controla la cacería de subsistencia a favor de las comunidades, sin embargo desconoce la presión de caza que esta actividad ejerce sobre las poblaciones de mamíferos. En ese sentido, la ausencia de esta información motivó a realizar el presente trabajo, el cual contribuirá a cimentar las bases para la formulación de futuros programas de conservación que conlleven al aprovechamiento sostenible de los mamíferos, beneficiando a las comunidades que usan el recurso y por consiguiente los ecosistemas que alberga esta importante área.

\section{MATERIALES Y MÉTODOS}

\section{ÁREA DE ESTUDIO}

El estudio se realizó en los sectores Alemán y Loboyacu pertenecientes a la Reserva Nacional Matsés ( $5^{\circ} 43^{\prime} 47.87^{\prime \prime} \mathrm{S}, 73^{\circ} 22^{\prime} 29.09^{\prime \prime} \mathrm{W}$ ) que cuenta con una superficie de 420,635.34 ha, ubicada en la región noreste de la Amazonía peruana, a 150 $\mathrm{km}$ al sureste de la ciudad de Iquitos. Está en los distritos de Requena, Soplin y Yaquerana, y en la provincia de Requena de la Región Loreto. Geográficamente se ubica entre las cuencas de los ríos Yavarí y el bajo Ucayali entre el interfluvio de los ríos Tapiche, Blanco, Gálvez y Yaquerana, limitando al oeste con las cabeceras del río Gálvez, a unos $3 \mathrm{~km}$ del río Blanco. Al sur colinda con el Parque Nacional Sierra del Divisor, al este con la Comunidad Nativa Matsés.

El Sector Alemán, comprende los márgenes de la quebrada Alemán (afluente del río Tapiche), abarcando bosques de colinas bajas fuertemente disectadas, terrazas altas de ligera a moderadamente disectadas, terrazas altas de drenaje muy pobre y terrazas bajas de drenaje imperfecto a pobre. En este sector los moradores de la comunidad campesina "Los Cuatro Hermanos del Alemán", realizan el aprovechamiento de fauna. Esta comunidad se encuentra asentada en la zona de amortiguamiento de la Reserva y está conformada por cuatro anexos denominados Monte Sinaí (70 familias aproximadamente), Alfa \& Omega (12), Concordancia (20) y Nuevo Salvador (12). Mientras que el sector Loboyacu se ubica margen derecha de la quebrada Loboyacu y la margen izquierda de la parte media del río Gálvez, ocupando bosques de colinas bajas fuertemente disectadas y terrazas bajas de drenaje imperfecto a pobre. En este sector, la Comunidad Nativa Matsés es la única que aprovecha los recursos naturales, siendo San Mateo (11), Jorge Chávez (11), Remoyacu (22) y Buen Perú (19) los anexos más cercanos a este lugar, y los que registran mayor ingreso al área para el aprovechamiento de recursos naturales con fines de subsistencia (Figura $1)$.

\section{MÉTODOS}

La información se obtuvo de los registros de caza, también llamado como autorización de aprovechamiento de los cazadores, archivados por el personal guardaparque de la Reserva en los puestos de vigilancia Alemán y Loboyacu, entre el período de enero del 2014 a diciembre del 2015, con el fin de obtener el promedio anual de individuos y biomasa extraída.

Datos adicionales como el área de caza, estrategias y herramientas que usan los cazadores, se obtuvo utilizando las consideraciones señaladas por Fang et al., (2008), quienes usan una combinación de metodologías participativas. Se utilizó las siguientes metodologías:

a) Mapas participativos: fueron reuniones con los cazadores, a quienes se les brindó un mapa satelital del área tamaño A0, donde ubicaron lugares de acceso, campamentos y rutas usados para la cacería por los cuerpos de agua y al interior del bosque; información importante que sirvió para estimar el área de caza. 


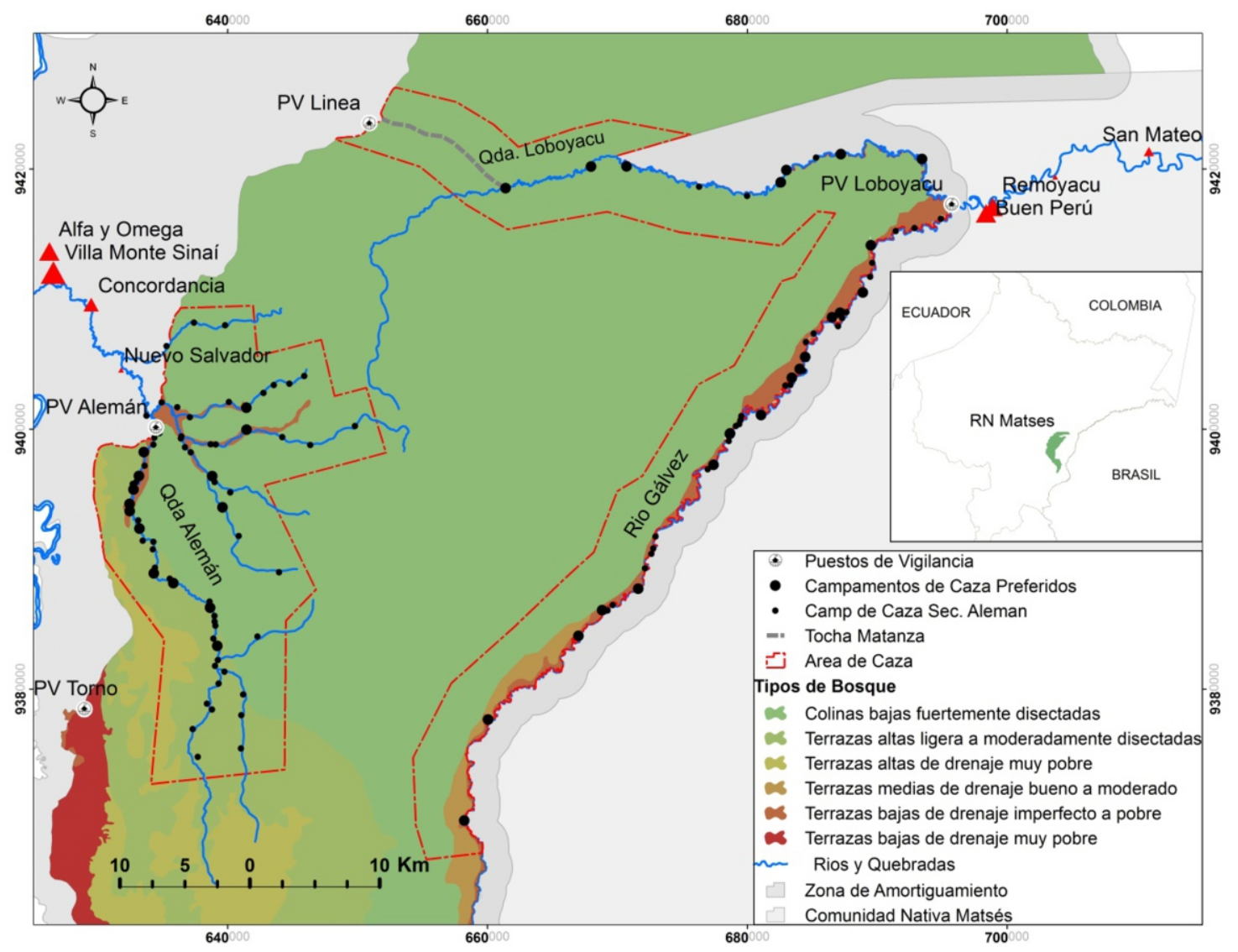

Figura 1. Área de caza del sector Alemán y Loboyacu de la Reserva Nacional Matsés, 2015.

b) Entrevistas Semiestructuradas: fueron entrevistas realizadas a los cazadores de manera individual, realizando preguntas de acuerdo a una guía o formulario previamente preparado, en algunos casos se requirió la ayuda de un traductor como en el sector Loboyacu, debido a que toda la población pertenece a la etnia Matsés.

Esta metodología permitió conocer los sitios de caza preferidos, las estrategias y herramientas de caza.

c) Diálogos Interactivos: fueron los intercambios espontáneos de información con los cazadores, aprovechando algunas faenas cotidianas como comidas, visitas, encuentros casuales, mingas y reuniones comunales que ayudaron a conocer datos relacionados a la cacería de mamíferos y sus perspectivas de conservación de algunas especies de mamíferos.

\section{ANÁLISIS DEDATOS}

Con ayuda del programa ArcGis 10.2, y usando los diferentes sitios que los cazadores indicaron en el mapa satelital, como campamentos y/o rutas de cacería, se estimó el área de caza.

El total de individuos cazados entre los años 2014-2015 se obtuvo de los registros de caza de Alemán y Loboyacu, posteriormente se obtuvo el índice de presión de caza (ind $/ \mathrm{km}^{2}$ ), dividiendo el promedio anual de individuos cazados entre el área de caza estimada. Adicionalmente, para determinar la diferencia anual de individuos extraídos por especie en cada sector, se usó la prueba estadística no paramétrica de muestras relacionadas de Wilcoxon, y para comparar la diferencia de individuos extraídos por especie entre los sectores se usó la prueba no paramétrica de muestras independientes de Mann-Whitney. 

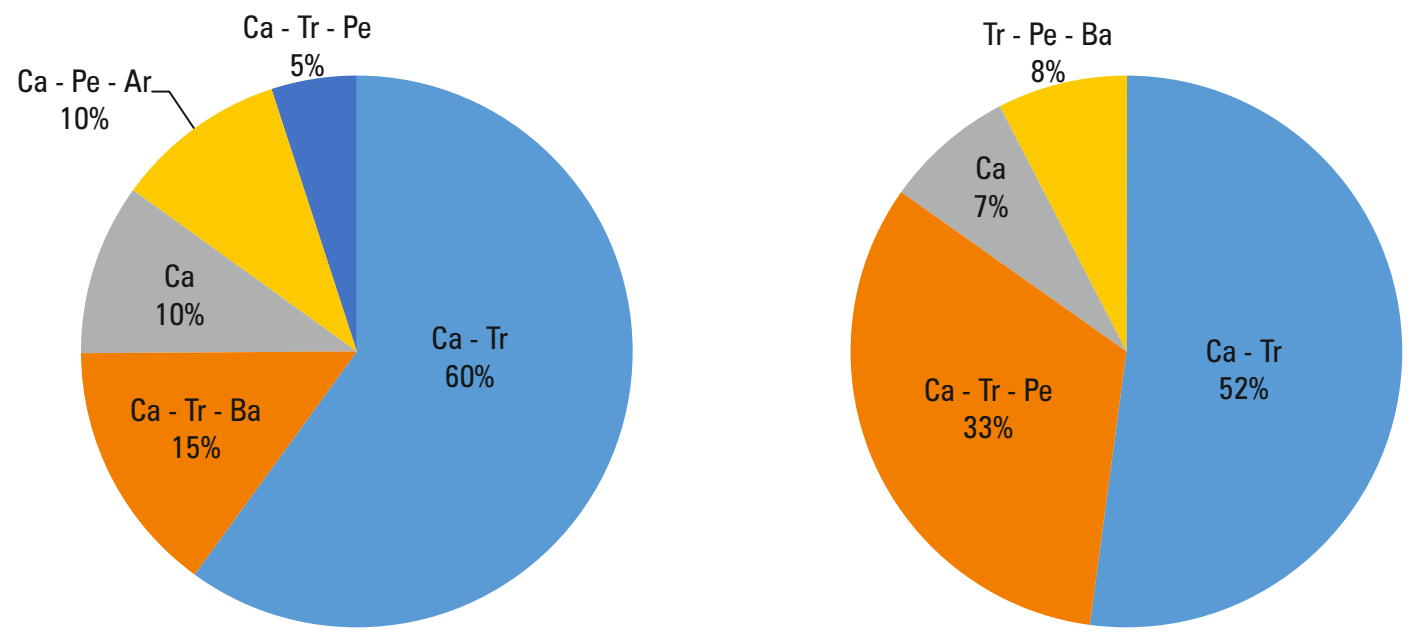

Figura 2. Estrategias de caza empleadas en el sector Alemán (a) y Loboyacu (b) de la Reserva Nacional Matsés, 2015. Ca: Canoa; Tr: Trocha; Ba: Barbacoa; Pe: Perro, Ar: Armadillo.

\section{RESULTADOS}

\section{ÁREADECAZA}

El área de caza estimada para el sector Alemán fue de $452 \mathrm{~km}^{2}$, calculada en base a 70 campamentos, de los cuales sólo 14 son utilizados para establecerse y el resto son usados como campamentos provisionales. De todos los campamentos, diez están distribuidos en la quebrada Alemán desde el Puesto de Vigilancia hasta la parte alta, en la intersección con la quebrada Almendra; cuatro campamentos están ubicados en la quebrada Blanco y sus tributarios. Asimismo, tres campamentos provisionales ubicados en la quebrada Pindayo también fueron considerados para la estimación del área de caza, que antes del presente estudio no se conocía la existencia de estos campamentos (Figura $1)$.

Para el sector Loboyacu, se estimó un área de caza de $497 \mathrm{~km}^{2}$, en base a 59 campamentos, de los cuales 22 son utilizados para establecerse y el resto son usados como campamentos provisionales. De todos los campamentos, 15 de los 22 , se distribuyen en el río Gálvez abarcando desde el Puesto de Vigilancia Loboyacu hasta la quebrada Sencö. En la quebrada Loboyacu están ubicados 7 campamentos, desde su desembocadura hasta el campamento Casa Matanza. También se tomó en cuenta la Trocha Matanza, que es usada por los pobladores Matsés para dirigirse a la ciudad de Requena, los cuales aprovechan los dos días de caminata para realizar la cacería (Figura 1).

\section{ESTRATEGIAS DE CAZA Y HERRAMIENTAS DE CAZA}

Las estrategias de caza se agruparon en cuatro categorías: búsqueda, espera, trampeo y casual; dentro de "búsqueda" se encuentran la realizada por canoa, trocha y utilizando perros, las dos primeras consisten en recorridos diurnos y nocturnos por los cuerpos de agua y al interior del bosque, mientras que la realizada usando perros, consiste en acorralar a Dasyprocta fuliginosa, Myoprocta pratti, Nasua nasua y los pecaríes; dentro de la categoría "espera", figura únicamente la cacería utilizando barbacoas, que consiste en preparar una plataforma con troncos y ramas cerca de una colpa en donde se espera la llegada de un animal. Dentro de la categoría "trampeo", figura sólo el armadillo, que consiste en utilizar un cañón de acero, ubicándose en la parte posterior un cartucho, un percutor y una cuerda que se activa con el paso del animal. Finalmente la categoría "casual", consiste en el encuentro oportuno de animales, cuando los pobladores realizan otras actividades de aprovechamiento, como la extracción de hoja de Lepidocaryum tenue "Irapay", la pesca y actividades comunes en estos lugares.

Como resultado de la entrevista a 20 cazadores del sector Alemán, la categoría búsqueda fue la más usada y la combinación de las estrategias canoa trocha obtuvo el mayor porcentaje $(60 \%)$, seguido de canoa - trocha - barbacoa con $15 \%$ y las estrategias sólo por canoa y canoa - perro armadillo obtuvieron $10 \%$ cada una y la canoa trocha-perro alcanzó 5\% (Figura 2). 


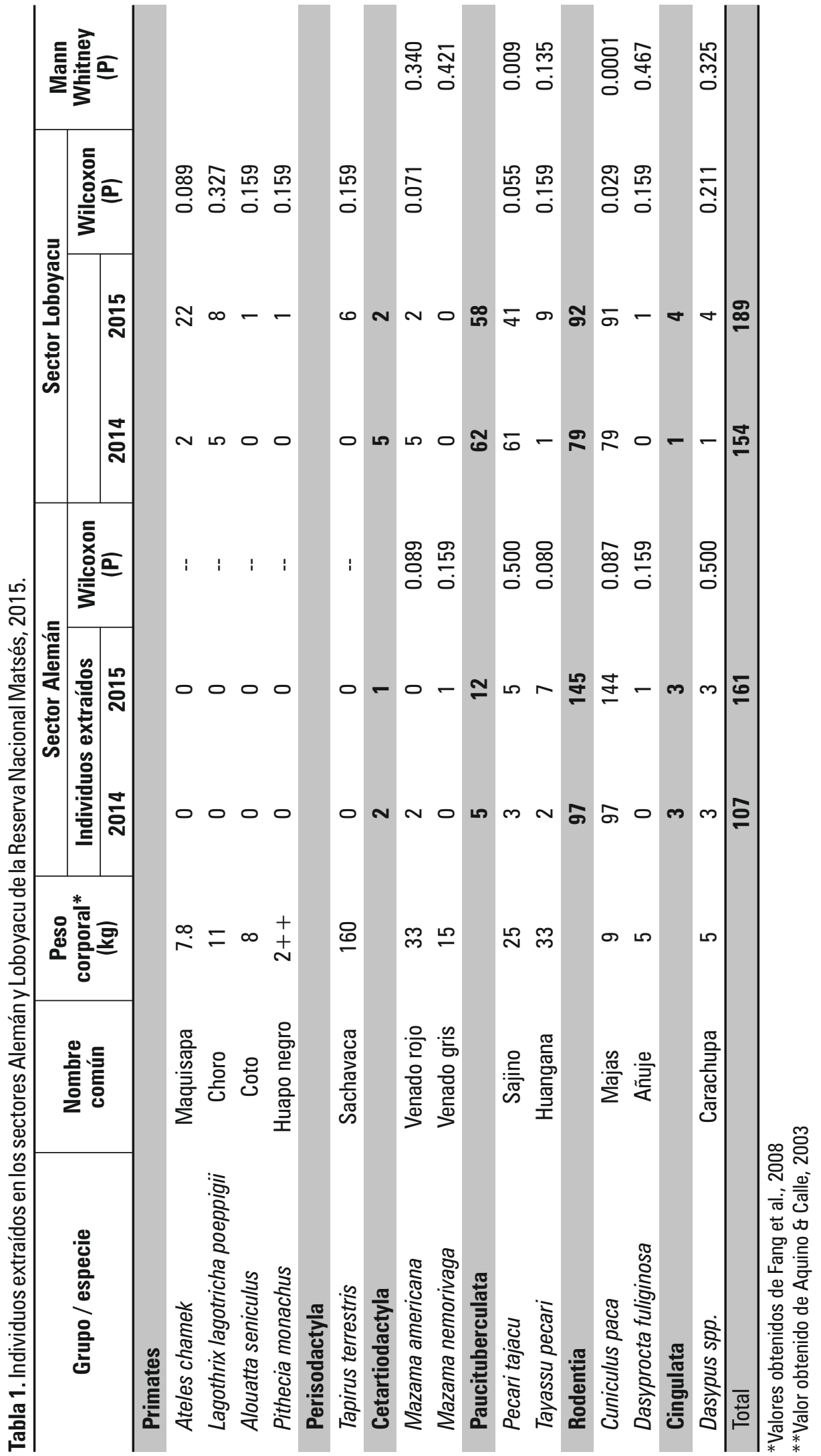


Tabla 2. Presión de caza en los sectores Alemán y Loboyacu de la Reserva Nacional Matsés durante el año 2015.

\begin{tabular}{l|c|c|c}
\hline \multirow{2}{*}{ Grupo / especie } & \multirow{2}{*}{ Nombre común } & \multicolumn{2}{c}{ Presión de caza } \\
\cline { 3 - 4 } & & $\begin{array}{c}\text { Sector Alemán } \\
(\mathbf{4 5 2} \mathbf{~ k m 2 )}\end{array}$ & $\begin{array}{c}\text { Sector Loboyacu } \\
\text { (492 } \mathbf{~ k m 2 )}\end{array}$ \\
\hline Primates & Maquisapa & $\mathbf{0 . 0 0 0}$ & $\mathbf{0 . 0 4 0}$ \\
Ateles chamek & Choro & 0.000 & 0.024 \\
Lagothrix lagotricha poeppigii & Coto & 0.000 & 0.014 \\
Alouatta seniculus & Huapo negro & 0.000 & 0.002 \\
Pithecia monachus & & 0.000 & 0.002 \\
Perisodactyla & Sachavaca & $\mathbf{0 . 0 0 0}$ & $\mathbf{0 . 0 0 6}$ \\
Tapirus terrestris & & 0.000 & 0.006 \\
Cetartiodactyla & Venado rojo & $\mathbf{0 . 0 0 4}$ & $\mathbf{0 . 0 0 8}$ \\
Mazama americana & Venado gris & 0.002 & 0.008 \\
Mazama nemorivaga & & 0.002 & 0.000 \\
\hline Paucituberculata & Sajino & $\mathbf{0 . 0 2}$ & $\mathbf{0 . 1 1 5}$ \\
Pecari tajacu & Huangana & 0.009 & 0.105 \\
Tayassu pecari & & 0.011 & 0.010 \\
\hline Rodentia & Majas & $\mathbf{0 . 2 7}$ & $\mathbf{0 . 1 7 3}$ \\
Cuniculus paca & Añuje & 0.268 & 0.171 \\
Dasyprocta fuliginosa & & 0.002 & 0.002 \\
Cingulata & Carachupa & $\mathbf{0 . 0 0 7}$ & $\mathbf{0 . 0 0 6}$ \\
Dasypus spp. & & 0.007 & 0.006 \\
\hline Total & & $\mathbf{0 . 3 0 1}$ & $\mathbf{0 . 3 5 2}$ \\
\hline & & & \\
\hline
\end{tabular}

En cuanto a las herramientas de caza utilizadas por los cazadores del sector Alemán, se destaca el uso de escopeta, complementado con el uso de machete y el arpón. Mientras que en el sector Loboyacu la entrevista fue realizada a 15 cazadores Matsés, resultando la categoría búsqueda la más usada y la combinación de las estrategias canoa trocha obtuvo el mayor porcentaje (52\%), seguido de canoa-trocha-perro con $33 \%$, trocha - perro barbacoa con $8 \%$ y únicamente canoa con $7 \%$ (Figura 2). Entre las herramientas de caza utilizadas por los cazadores de este sector, se destaca también el uso de la escopeta, complementado con el uso del arco y el machete. Aunque el uso del arco/flecha, sólo es usado por algunos de los cazadores más experimentados, en su mayoría ancianos.

\section{INDIVIDUOS EXTRAÍDOS}

Entre el periodo 2014-2015, se extrajeron 611 individuos de mamíferos en Alemán y Loboyacu, correspondientes a 12 especies, distribuidos en seis órdenes. En ambos sectores Cuniculus paca fue la especie más cazada, siendo el sector Alemán la que presentó mayor cantidad de individuos extraídos, la cacería de esta especie predomina sobre las otras que son cazadas de forma esporádica en este sector. Mientras que en el sector Loboyacu la cacería de esta especie se alterna con Pecari tajacu quien es la segunda especie más cazada, adicionalmente se reporta la cacería de primates y Tapirus terrestris (Tabla 1). En conjunto hay un índice de presión de caza de 0.301 individuos $/ \mathrm{Km}^{2}$ y 0.352 Individuos $/ \mathrm{Km}^{2}$ para Alemán y Loboyacu respectivamente (Tabla 2).

Al comparar los individuos extraídos por especie entre los años 2014-2015 en cada sector, resultó que no existe diferencias en ninguna de las especies en el sector Alemán, mientras que para el sector Loboyacu, Cunículus paca $(\mathrm{p}=0.029)$, fue la única que presentó diferencia, debido a que en el 2015 se extrajo mayor cantidad de individuos. Finalmente, la comparación de los individuos extraídos de especie entre sectores, indicó diferencias significativas para Pecari tajacu $(\mathrm{p}=0.009)$ y Cuniculus paca $(\mathrm{p}=$ 0.0001 ), las cuales fueron las especies más cazadas en Loboyacu y Alemán, respectivamente (Tabla 1). 


\section{DISCUSIÓN}

En ambos sectores de caza, 12 especies de mamíferos resultaron entre los preferidos por los cazadores, 11 de las cuales se cazaron en el sector Loboyacu y siete en el sector Alemán, la diferencia con respecto al menor número de especies cazadas en Alemán, podría estar relacionada a los acuerdos establecidos entre la comunidad campesina "Los Cuatro Hermanos del Alemán" y la Jefatura de la Reserva, donde se restringe el aprovechamiento de especies susceptibles a la caza, entre ellas los primates y Tapirus terrestris, especies consideradas amenazadas, así como aquellas de importancia para el turismo entre ellas Panthera onca, Pteronura brasiliensis, Ara macao, Harpia harpyja, Cacajo calvus (SERNANP, 2014). A pesar de los acuerdos existentes en este sector, se observó un cráneo de $T$. terrestris en el campamento "Rurón", evidenciando que sigue cazándose esta especie en el sector, quizás por personas de la comunidad o ajenas a ella. Por otro lado, en el sector Loboyacu, no existe ningún acuerdo hasta la fecha, por lo tanto, los pobladores de la Comunidad Nativa Matsés continúan aprovechando tradicionalmente los primates y $T$. terretris, y otras especies de fauna que encuentran casualmente.

Las cifras correspondientes al número de especies aprovechadas en Loboyacu, resultaron inferiores a otros lugares donde la cacería no es regulada, para el Perú, específicamente en el departamento de Loreto como en el río Algodón (Aquino et al., 2007a), cuenca alta del río Itaya (Aquino et al., 2007b) y la comunidad de Bretaña (Saldaña \& Saldaña, 2011); en Bolivia, la comunidad indígena Akae (Cuéllar, 2000); en Ecuador, el área de amortiguamiento del Parque Nacional Yasuní (Cueva et al., 2004); en Colombia, la Comunidad Zancudo (Tafur, 2010), y en México, la Selva Zoque (Lira-Torres et al., 2014). Las diferencias del número de especies aprovechadas en cada uno de estos lugares, podría deberse a las diferentes cosmovisiones que tienen las personas con respecto al aprovechamiento de la fauna. Como es el caso de algunos pobladores de la Comunidad Matsés, argumentando que no cazan ciertas especies como Sapajus macrocephalus y Cebus unicolor, porque su consumo puede generar un comportamiento "holgazán" del individuo, y si consumieran Hydrochaeris hydrochaeris puede provocar alergias en la piel.

Los 268 individuos extraídos, tanto en el sector Alemán como los 343 individuos en Loboyacu, son inferiores a los referidos en investigaciones realizadas en los bosques del Área de Conservación Regional Comunal Tamshiyacu-Tahuayo, donde se cazaron 1235 individuos (Puertas, 1999), y también en la cuenca alta del río Itaya, donde se extrajo 1176 individuos (Aquino et al., 2007b).

Algunos factores que podrían estar influyendo en la diferencia de los resultados de la presente investigación con los estudios realizados, sería el tipo de caza (subsistencia) y la cantidad de familias beneficiarias en relación a otros lugares, como es el caso del Área de Conservación Regional Comunal Tamshiyacu-Tahuayo (Puertas, 1999) y la cuenca alta del río Itaya (Aquino et al., 2007b). Otro de los factores que explicarían el limitando el número de individuos de la presente investigación, serían los datos parciales registrados en las fichas de aprovechamiento de recursos naturales, donde sólo se registra la fauna procedente de la Reserva, y se excluye a las especies y el número de individuos cazados alrededor de sus comunidades. Es oportuno mencionar que, en algunas épocas de festividades, como las "Fiestas Patrias en el mes de julio" y "Navidad en el mes de diciembre", se incrementa el número de cazadores que ingresan a la Reserva, y por ende el número de individuos cazados, por tal razón la cacería no es constante a lo largo del año.

Dentro de las especies cazadas en el sector Alemán, Cuniculus paca fue la más extraída con 241 individuos, resultado que evidenciaría la preferencia de los cazadores hacia la especie, por la calidad de su carne, y el poco esfuerzo que demanda su cacería mediante canoa; razones que explicarían que su cacería es dirigida. En el sector Loboyacu, C. paca, también fue la especie más preferida por los cazadores Matsés, con 170 individuos extraídos, esta diferencia con respecto al sector Alemán, estaría relacionada al tipo de estrategia de caza que utilizan los Matsés, quienes prefieren cazar usando trochas durante el día, lo cual les permite cazar pecaríes, primates y otras especies. Los resultados de preferencia sobre esta especie, coinciden con las investigaciones realizadas en cuenca alta del río Itaya (Aquino et al., 2007b), los bosques aledaños a las comunidades San Miguel y Parinari de la Reserva Nacional Pacaya-Samiria (Aquino \& Calle, 2003), comunidad de Bretaña (Saldaña \& Saldaña, 2011), Comunidad Nativa Mushuckllacta de Chipaota de la zona de amortiguamiento del Parque Nacional Cordillera Azul (Sánchez \& Vásquez, 2007) y zona de amortiguamiento del Parque Nacional YasuníEcuador (Cueva et al., 2004).

De las cuatro categorías utilizadas en la caza de mamíferos en el sector Alemán y Loboyacu, la categoría búsqueda fue la más utilizada en ambos sectores, la cual incluye a las estrategias de caza realizadas por trocha, canoa, con perro y combinaciones de estas, esta categoría también ha 
sido utilizada por otras comunidades de la zona de amortiguamiento del Parque Nacional YasuníEcuador (Cueva et al., 2004) y la Comunidad Zancudo-Colombia (Tafur, 2010), al parecer esta categoría resulta más efectiva, que la de esperar o construir trampas para capturar a los animales. El mayor uso de algunas estrategias de caza, podría influir en la preferencia de los cazadores hacia ciertas especies, como se constató en los sectores evaluados, por ejemplo, los cazadores de Alemán y Loboyacu, prefirieron utilizar la estrategia de caza en canoa, y la especie preferida resultó Cuniculus paca, esto se evidencia en los resultados de presión de caza, donde esta especie obtuvo el mayor número de individuos cazados en ambos sectores. Entre las herramientas de caza más usadas, figura la escopeta, el cual ha sustituido las herramientas tradicionales (arco/flecha y la lanza) de los Matsés, que en la actualidad solamente son utilizadas por algunas personas, en su mayoría ancianos; esta tendencia de sustitución de armas tradicionales por la escopeta, no sólo se evidencia en los Matsés, sino también en la mayoría de grupos humanos indígenas de otros lugares de la Amazonía, como los Kichwas del Pastaza (Escobedo et al., 2004), los Cashinahuas del río Curanga y Purús (Gil, 2004), los Tícuna y Sikuani de Colombia (Rangel, 2005; Bedoya, 1999) y los Kichwas de la zona de amortiguamiento del Parque Nacional Yasuní de Ecuador (Cueva et al., 2004), según las referencias, la escopeta es un arma que resulta más efectiva y facilita la cacería y resulta más fácil aprender a manejar un arma, que adiestrarse en el uso y manejo de armas tradicionales, manifiestan los Matsés.

\section{CONCLUSIÓN}

Los aspectos evaluados de la presión de caza en la Reserva Nacional Matsés, demuestran que la cacería es moderada. Esto quiere decir, que los acuerdos comunales establecidos en el sector Alemán, están actuando bien al minimizar el impacto de la cacería sobre los mamíferos, ya que limitan el número de individuos cazados, prohíben especies vulnerables, y controlan el uso de herramientas y estrategias. Se resalta la cacería de subsistencia en el sector Loboyacu, donde los acuerdos comunales no existen, y todavía algunos pobladores practican la cacería de forma tradicional usando herramientas y estrategias ancestrales.

\section{BIBLIOGRAFÍA CITADA}

Aquino, R.; Calle, A. 2003. Evaluación del estado de conservación de los mamíferos de caza: un modelo comparativo en comunidades de la
Reserva Nacional Pacaya Samiria (Loreto, Perú). Revista Peruana de Biología, 10(2): 163-174.

Aquino, R.; Pacheco, T.; Vásquez, M. 2007a. Evaluación y valorización económica de la fauna silvestre en el río Algodón, Amazonía peruana. Revista Peruana de Biología, 14 (2): 187-192.

Aquino, R.; Terrones, C.; Navarro, R.; Terrones, W. 2007b. Evaluación del impacto de la caza en mamíferos de la cuenca del río Alto Itaya, Amazonía peruana. Revista Peruana de Biología, 14 (2): 181-186.

Bedoya, M. 1999. Patrones de cacería en una Comunidad Indígena Tícuna en la Amazonía Colombiana. In: Tula Fang, Olga Montenegro y Richard Bodmer (Eds). Manejo y conservación de fauna silvestre en América Latina. p. 71-75. La Paz-Bolivia: Instituto de Ecología.

Bodmer, R.; Allen, C.; Penn, J.; Aquino, R.; Reyes, C. 1999. Evaluación del uso sostenible de la fauna silvestre en la Reserva Nacional Pacaya Samiria. The Nature Conservancy.

Cuéllar, R.L. 2000. Aprovechamiento de la fauna silvestre en una comunidad de agricultores: Los Guaraníes de Akae, Santa Cruz, Bolivia. In: Cabrera, E.; Mercolli, C.; Resquín, R (Eds). Manejo y conservación de fauna silvestre en América Latina. p. 147-157.

Cueva, R.; Ortiz, A.; Jorgenson, J.P. 2004. Cacería de fauna silvestre en el área de amortiguamiento del Parque Nacional Yasuní, Amazonía Ecuatoriana. Manejo y conservación de fauna silvestre en América Latina. p. 524-539.

Escobedo, A.; Ríos, C.; Bodmer, R.; Puertas, P.E. 2004. La caza de animales silvestres por los Kichwas del río Pastaza, Nor-Oriente Peruano: Iniciativas de manejo comunal. Manejo y conservación de fauna silvestre en América Latina. p. 479-488.

Fang, T.G.; Bodmer, R.; Puertas, P.E.; Mayor, P.; Pérez-Peña, P.E.; Acero R.; Hayman, D. 2008. Certificación de pieles de pecaries en la Amazonía Peruana: Una estrategia para la conservación y manejo de la fauna silvestre en la Amazonía peruana. Wust Ediciones, Lima. 203pp.

Gil, J.G. 2004. Aprovechamiento de la fauna silvestre en comunidades Cashinahua del río Curanja y Purús: Informe Técnico. 30pp.

Hurtado-Gonzales, J.L.; Bodmer, R.E. 2004. Assessing the sustainability of brocket deer hunting in the Tamshiyacu-Tahuayo Communal Reserve, Northeastern Peru. Biological Consevation, 116: 1-7. 
Lira-Torres, I.; Briones-Salas, M.; Ojeda-Ramírez, F.R.; Peláez, D. 2014. Uso y aprovechamiento de la fauna silvestre en la Selva Zoque, México. Acta Zoológica Mexicana (nueva serie), 30 (1): 70-90.

Ojasti J. 1993. Utilización de la fauna silvestre en América Latina: Situación y perspectivas para un manejo sostenible. Organización de las Naciones Unidas para la Agricultura y la Alimentación. Roma-Italia. 261pp.

Pérez-Peña, P. 2011. Evaluación poblacional de mamíferos y aves de caza en la Concesión de Conservación Lago Preto Paredón y en cuenca media del Yavarí Mirín. Reporte final. Iquitos. $31 \mathrm{pp}$.

Pérez-Peña, P.; Gonzales-Tanchiva, C.; TrigosoPinedo, M. 2016. Evaluación del plan de manejo de animales de caza en la Reserva Nacional Pucacuro. Folia Amazónica, 25 (1): 1 - 16.

Puertas, P.E. 1999. Hunting effort analysis in northeastern Peru: the case of the Reserva Comunal Tamshiyacu-Tahuayo. Florida. Tesis de Maestría, Universidad de Florida. Florida, EE.UU.

Rangel A.M. 2005. Importancia de la fauna silvestre en la etnia Sikuani, Comunidad de Cumarianae, Selva de Matavén, Vichada, Colombia.
SERNANP. 2014. Plan Maestro de la Reserva Nacional Matsés 2014 - 2019. Servicio Nacional de Áreas Naturales Protegidas, Lima. 94pp.

Saldaña, J.S.; Saldaña, V.L. 2011. La cacería de animales silvestres en la comunidad de Bretaña, río Puinahua, Loreto-Perú. Revista Colombiana Ciencia Animal, 3 (2): 225-237.

Sánchez, A.; Vásquez, P. 2007. Presión de caza de la comunidad nativa Mushuckllacta de Chipaota, zona de amortiguamiento del Parque Nacional Cordillera Azul, Perú. Ecología Aplicada, 6 $(1,2): 131-138$.

Tafur M.D.P. 2010. Evaluación de la sostenibilidad de la cacería de mamíferos en la Comunidad de Zancudo, Reserva Nacional Natural Puinawai, Guainía-Colombia. Tesis de Maestría, Universidad Nacional de Colombia. Bogotá, Colombia.

Vriesendorp, C.; Pitman, N.; Rojas, J.I.; Pawlak, B.A.; Calixto, L.; Vela, M.; Fasabi, P. 2006. Matses Rapid Biological Inventories Report 16. The Field Museum. Chicago, Illinois.

Recibido: 20 de Febrero del 2017

Aceptado para publicación: 28 de Abril del 2017 
